\title{
Chloroplast proteome analysis of Nicotiana tabacum overexpressing TERF1 under drought stress condition
}

\author{
Wei Wu and Yanchun Yan ${ }^{*}$
}

\begin{abstract}
Background: Chloroplast is indispensable for plant response to environmental stresses, growth and development, whose function is regulated by different plant hormones. The chloroplast proteome is encoded by chloroplast genome and nuclear genome, which play essential roles in plant photosynthesis, metabolism and other biological processes. Ethylene response factors (ERFs) are key transcription factors in activating the ethylene signaling pathway and plant response to abiotic stress. But we know little about how ethylene regulates plastid function under drought stress condition. In this study we utilized tobacco overexpressing tomato ethylene responsive factor 1 (TERF1), an ERF transcription factor isolated from tomato, to investigate its effects on the plastid proteome under drought stress condition by method of iTRAQ technology.

Results: Results show that TERF1 represses the genes encoding the photosynthetic apparatus at both transcriptional and translational level, but the genes involved in carbon fixation are significantly induced by TERF1. TERF1 regulates multiple retrograde signaling pathways, providing a new mechanism for regulating nuclear gene expression. TERF1 also regulates plant utilization of phosphorus ( $\mathrm{Pi}$ ) and nitrogen $(\mathrm{N})$. We find that several metabolic and signaling pathways related with Pi are significantly repressed and gene expression analysis shows that TERF1 significantly represses the Pi transport from root to shoot. However, the $\mathrm{N}$ metabolism is upregulated by TERF1 as shown by the activation of different amino acids biosynthesis pathways due to the induction of glutamine synthetase and stabilization of nitrate reductase although the root-to-shoot $\mathrm{N}$ transport is also reduced. TERF1 also regulates other core metabolic pathways and secondary metabolic pathways that are important for plant growth, development and response to environmental stresses. Gene set linkage analysis was applied for the upregulated proteins by TERF1, showing some new potential for regulating plant response to drought stress by TERF1.

Conclusions: Our research reveals effects of ethylene signaling on plastid proteome related with two key biological processes, including photosynthesis and nutrition utilization. We also provide a new mechanism to regulate nuclear gene expression by ERF1 transcription factor through retrograde signals in chloroplast. These results can enrich our knowledge about ERF1 transcription factor and function of ethylene signaling pathway.
\end{abstract}

Keywords: Chloroplast proteome, Drought stress, TERF1, Ethylene signaling pathway

\footnotetext{
*Correspondence: yanyanchun@caas.cn

Graduate School of Chinese Academy of Agricultural Sciences, No. 12

Zhongguancun South St., Haidian District, Beijing 100081, People's
}

Republic of China 


\section{Background}

Ethylene is a gaseous phytohormone that plays vital roles in plant growth, development as well as response to abiotic and biotic stresses. ERF1 protein is a key transcription factor for activating the ethylene signaling pathway by binding the GCC box and other cis-elements in the promoters of ethylene-responsive genes, which is an ideal candidate gene for improving plant tolerance of different abiotic stresses (Kavas et al. 2015).

Chloroplast is an organelle derived from the cyanobacteria and its proteome is encoded by nuclear genome and its own genome. Apart from the key function of photosynthesis, chloroplast is also responsible for the biosynthesis of different metabolites, including amino acids, vitamins, fatty acids, nucleotides, phytohormones as well as assimilation of sulfur and nitrogen (Daniell et al. 2016). Chloroplast has been found to regulate plants' response to different environmental stresses through organellar signaling pathways because it can act as environment sensor (Kmiecik et al. 2016).

At transcriptional level different phytohormones have been found to regulate chloroplast transcription. Abscisic acid (ABA) represses the plastid encoded RNA polymerase (PEP) and nuclear encoded RNA polymerase (NEP), leading to the reduced transcript level of most chloroplast genes, which can be minimized by cytokinin (Yamburenko et al. 2015). Methyl jasmonate (MeJA), gibberellic acid [GA(3)], an auxin (indole-3-acetic acid, IAA) repress both transcription and transcript accumulation of chloroplast genes, while a brassinosteroid (24-epibrassinolide, BR) can counteract these effects (Zubo et al. 2011).

Additionally, characterization of chloroplast proteome changes under environmental stress condition attracted the attention of the researchers. 31 chloroplast proteins were found to be differentially regulated by drought stress, which are mainly enriched in the Gene Ontology (GO) category of "energy" (Zubo et al. 2011). Kosmala et al. identified 10 differentially accumulated chloroplast proteins in high-drought-tolerant and low-droughttolerant of Festuca arundinacea (Kosmala et al. 2012). Uberegui et al. revealed the function of Executer proteins in plant chloroplast response to increased light condition (Uberegui et al. 2015). Wang et al. studied the chloroplast proteome changes in mangrove under different salt stress conditions (Wang et al. 2013). Although these studies have revealed some mechanisms in chloroplast response to environmental stresses, most of these proteomics studies utilized 2D-Gel method, which restricted coverage of the chloroplast proteome and the accuracy of protein quantification. Additionally, there is little report on how ethylene regulate chloroplast proteome under environmental stress conditions.
TERF1, an ERF1 protein isolated from tomato, can activate ethylene signaling pathway through binding the GCC box and dehydration responsive element (DRE) in the promoters of the target genes (Huang et al. 2004). Overexpression of TERF1 can significantly improve tobacco tolerance of abiotic stress (Huang et al. 2004), however the detailed mechanism of TERF1 in improving the plant tolerance of drought stress is not clear. In this study we compared the chloroplast proteome between WT tobacco and tobacco overexpressing TERF1 under natural dehydration condition by the isobaric tags for relative and absolute quantification (iTRAQ) method, aiming to further reveal the function of TERF1 and ethylene in plant response to drought stress.

\section{Results}

TERF1 significantly changes the chloroplast proteome under drought stress condition

After drought stress was exerted we measured the relative water content (RWC) of the WT and transgenic tobacco and results showed that the RWC of WT tobacco decreased faster than the transgenic ones after 10 days of dehydration and at 20 days of dehydration the RWC of WT tobacco decreased by more than $35 \%$ and the TERF 1 tobacco decreased only about 18\% (Additional file 1: Figure S1), indicating that TERF1 significantly reduced water loss under drought stress condition. We compared the chloroplast proteome between TERF1 and WT tobacco after 20 days of dehydration when the RWC of WT tobacco was significantly lower than TERF1 tobacco.

We identified 13,049 unique peptides (Additional file 2: Table S9), which were searched against the UniProt database of Nicotiana tabacum and 4732 proteins were identified and quantified (at least two unique peptide with high confidence), among which 4694 proteins are encoded by nuclear genome (Additional file 3: Table S2) and 38 proteins are encoded by chloroplast genome (Additional file 9: Table S7). A significant difference is observed in chloroplast proteome between WT and TERF1 tobacco supported by principal component analysis (PCA) (Additional file 4: Figure S2).

We use more than 1.20 -fold $(\mathrm{P}<0.05)$ or less than 0.83 -fold $(\mathrm{P}<0.05)$ cutoff to identify the differentially expressed proteins (DEPs), of which 189 proteins are significantly induced (Additional file 5: Table S3) and 273 proteins are significantly repressed (Additional file 6: Table S4). In order to dissect the function of the DEPs the KEGG pathway enrichment analysis was applied and the significantly enriched pathways are shown in Tables 1 and 2. The results of gene ontology (GO) enrichment analysis for the DEPs are listed in Additional file 7: Table S5 and Additional file 8: Table S6, respectively. 
Table 1 KEGG pathway enrichment for the upregulated DCPs

\begin{tabular}{llll}
\hline Term & ID & Number of the enriched proteins (\%) & P-value \\
\hline Metabolic pathways & ko01100 & $61(32 \%)$ & 0.009696835 \\
Biosynthesis of secondary metabolites & ko01110 & $41(21 \%)$ & 0.005100797 \\
Biosynthesis of amino acids & ko01230 & $19(10 \%)$ & 0.002111965 \\
Terpenoid backbone biosynthesis & ko00900 & $5(3 \%)$ & 0.012298651 \\
Fatty acid biosynthesis & ko00061 & $5(3 \%)$ & $5(3 \%)$ \\
mRNA surveillance pathway & ko03015 & $4(2 \%)$ & 0.013993754 \\
Pantothenate and CoA biosynthesis & ko00770 & $4(2 \%)$ & 0.05547601 \\
Valine, leucine and isoleucine biosynthesis & ko00670 & $4(2 \%)$ & 0.007031075 \\
One carbon pool by folate & ko00460 & $4(2 \%)$ & 0.010395488 \\
Cyanoamino acid metabolism & ko00950 & $3(2 \%)$ & 0.014658739 \\
Isoquinoline alkaloid biosynthesis & ko00780 & 0.029672314 \\
Biotin metabolism & & $3(2 \%)$ & 0.021900026 \\
\hline
\end{tabular}

Table 2 KEGG pathway enrichment for the downregulated DCPs

\begin{tabular}{lllc}
\hline TERM & ID & Number of the enriched proteins (\%) & P-value \\
\hline Metabolic pathways & ko01100 & $62(23 \%)$ & 0.007587671 \\
Photosynthesis & ko00195 & $15(5 \%)$ & $1.66 \mathrm{E}-07$ \\
Photosynthesis-antenna proteins & ko00196 & $8(3 \%)$ & $1.22 \mathrm{E}-07$ \\
Glycerophospholipid metabolism & ko00564 & $6(2 \%)$ & 0.000985438 \\
Phenylpropanoid biosynthesis & ko00940 & $6(2 \%)$ & 0.007947381 \\
Phosphatidylinositol signaling system & ko04070 & $4(1 \%)$ & 0.004652457 \\
Ether lipid metabolism & ko00565 & $3(1 \%)$ & 0.002725805 \\
ABC transporters & ko02010 & $2(1 \%)$ & 0.005861127 \\
Diterpenoid biosynthesis & ko00904 & $2(1 \%)$ & 0.002013018 \\
\hline
\end{tabular}

\section{TERF1 exerts different effects on the expression} of the genes in photosynthetic light and dark reaction Chloroplast proteome are encoded by nuclear genome and chloroplast genome together, among which 98 proteins are encoded by tobacco chloroplast genome. The genes encoded by chloroplast genome are related with photosynthetic apparatus, the chloroplast genetic system, and other functions (Shimada and Sugiura 1991). 38 chloroplast genome encoded proteins were identified and quantified in our proteomics analysis, among which 27 proteins were repressed by TERF1 and most of these proteins are components of photosynthesis apparatus (Additional file 9: Table S7).

We also assessed the transcript level of the 79 genes encoded by chloroplast genome with confirmed function, showing that 61 genes were repressed and only 18 were induced by TERF1 (Additional file 10: Table S8). The significantly repressed genes encode subunits of PSI, PSII, NADH dehydrogenase, ATP synthase, cytochrome b6/f and ribosome proteins (Additional file 10: Table S8). As the bacteria-type $70 \mathrm{~S}$ ribosome is responsible for protein biosynthesis in chloroplast (Tiller and Bock 2014), the significant repression of the rps and $r p l$ genes by TERF1 contribute to the repression of protein biosynthesis in chloroplast.

Additionally TERF1 negatively regulates RNA editing in chloroplast as shown by the significant repression of Multiple Organellar RNA Editing Factor 8 (MORF8) (No. 122, Additional file 6: Table S4). MORF8 participates in chloroplast RNA editing as well as 8 mitochondrial genes editing (Glass et al. 2015; Huang et al. 2017). Repression of MORF8 will negatively affect chloroplast and plant growth and development (Glass et al. 2015).

TERF1 also represses many genes encoded by nuclear genome participating in the process of light harvesting, light reaction and photosynthetic electron transport (Additional file 8: Table S6). Additionally, we also found FtsH5, a thylakoid membrane-bound metalloprotease, was significantly repressed (No. 32, Additional file 6: Table S4), which will impair the D1 protein degradation and finally leads to photoinhibition (Kato et al. 2009). Finally, two genes involved in the chlorophyll 
biosynthesis, Glutamate-tRNA ligase (GluRSAt) and Protoporphyrinogen oxidase (PPO2) (No. 91 and No. 200, Additional file 6: Table S4), were also significantly repressed by TERF1. Repression of GluRSAt and PPO2 will reduce the accumulation of Glutamyl-tRNA ${ }^{\text {Glu }}$, starting substrate of tetrapyrroles biosynthesis, and Protoporphyrin IX, the last common precursor for chlorophyll and heme biosynthesis, respectively.

Although many genes involved in photosynthetic light reactions were repressed by TERF1, seven GO terms related with carbon fixation are significantly enriched for the upregulated DEPs, including "GO 0034637: cellular carbohydrate biosynthetic process", "GO 0033692: cellular polysaccharide biosynthetic process", "GO 0042732: D-xylose metabolic process", "GO 0009250: glucan biosynthetic process", "GO 0000271: polysaccharide biosynthetic process", "GO 0019252: starch biosynthetic process", GO 0005982: starch metabolic process" (Additional file 7: Table S5), indicating that carbon fixation in TERF1 tobacco is significantly upregulated.

According to the protein expression profile, several key genes involved in starch biosynthesis are significantly induced by TERF1. Protein Targeting To Starch (PTTS) is upregulated by more than $32 \%$ by TERF1, which localise granule-bound starch synthase to starch granules and is responsible for normal amylose synthesis (No. 125, Additional file 5: Table S3) (Seung et al. 2015). ADP-Glucose Pyrophosphorylase (ApL3), catalyzing the first and limiting step in starch biosynthesis (Sulmon et al. 2011), is significantly induced by $26 \%$ in TERF1 tobacco (No. 66, Additional file 5: Table S3). Starch Synthase 2 (SS2), involved in amylopectin biosynthesis (Zhang et al. 2008), is induced by more than $25 \%$ in TERF1 tobacco. The upregulation of the carbon fixation in TERF1 tobacco will also contribute to the repression of the genes involved in photosynthetic light reaction (Hausler et al. 2014).

\section{TERF1 regulates four key chloroplast metabolic pathways}

According to the KEGG pathway enrichment analysis, the pathway of "valine, leucine and isoleucine biosynthesis", "biosynthesis of amino acids" and "cyanoamino acid metabolism" are significantly enriched for the upregulated DEPs in TERF1 tobacco (Table 1). Another five GO terms related with amino acids metabolism (GO 1901607: alpha-amino acid biosynthetic process, GO 0006541: glutamine metabolic process, GO 0006545: glycine biosynthetic process, GO 0009070: serine family amino acid biosynthetic process, GO 0006556: S-adenosylmethionine biosynthetic process) are also significantly enriched for the upregulated DEPs (Additional file 7: Table S5), indicating that $\mathrm{N}$ assimilation is improved by TERF1 (Li et al. 2017).
Gene expression results show that TERF1 significantly represses NRT2.4 and NRT1.2 (Fig. 1a). NRT2.4 and NRT1.2 encode high-affinity and low-affinity nitrate transporter, respectively (Filleur et al. 2001), indicating that TERF1 restricts the nitrate uptake in the root under drought stress condition. Moreover, the significant induction of NLA in leaf will degrade NRT1.7 although NRT1.7 transcript level is slightly induced (Fig. 1b). NRT1.7 is responsible for source-to-sink nitrate remobilization and negatively regulates plant adaptation to limited nitrogen (Liu et al. 2017a, b), indicating that TERF1 also limits the $\mathrm{N}$ remobilization under drought stress condition.

However, at protein level we find that TERF1 significantly upregulates the glutamine synthetase (GS) (No. 67, Additional file 5: Table S3), a key enzyme for improving $\mathrm{N}$ use efficiency (NUE) through converting ammonium into glutamine (Wang et al. 2018). And two 14-3-3 proteins are significantly repressed by TERF1 (No. 2 and No. 3, Additional file 6: Table S4), which can stabilize nitrate reductase (NR), a key enzyme for reducing nitrate in chloroplast (Miller et al. 2008). $\mathrm{N}$ assimilation in chloroplast also correlates with the TCA cycle in the mitochondria because it can provide $\mathrm{C}$ skeleton for de novo ammonium assimilation. We find that the dicarboxylate transporter (No. 54, Additional file 5: Table S3) is significantly induced by TERF1, which can promote the transport of 2-oxoglutarate into the chloroplast, an important C skeleton for ammonium assimilation by GS (Taniguchi and Taniguchi et al. 2002). 2-oxoglutarate also regulates GS activity at transcriptional or post-translational level (Galvez et al. 1999). Upregulation of GS and stabilization of NR provide a possible mechanism for the enhanced $\mathrm{N}$

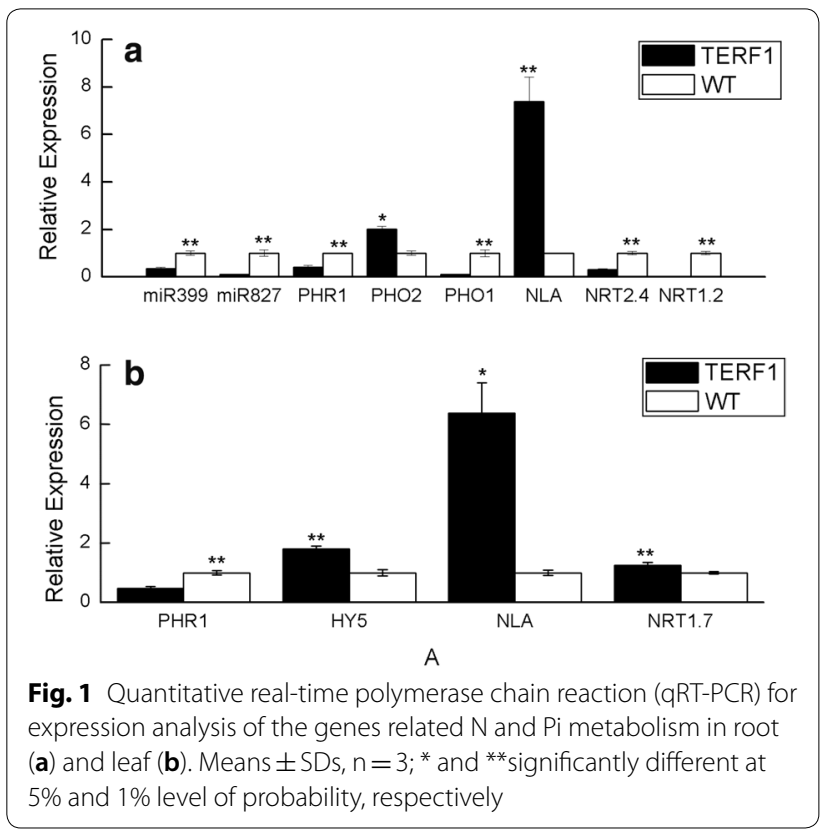


assimilation efficiency by TERF1. As NUE is composed of $\mathrm{N}$ uptake efficiecy and $\mathrm{N}$ utilization efficiency (Wang et al. 2018), we propose that TERF1 significantly improve $\mathrm{N}$ utilization efficiency.

As described above many genes related with photosynthesis are significantly repressed we speculate that TERF1 may repress Phosphorus $(\mathrm{Pi})$ utilization because $\mathrm{Pi}$ is an essential nutrient for plant photosynthesis (Zhang et al. 2014). Additionally we also find several pathways related with Pi metabolism or signaling pathway are significantly enriched for the downregulated proteins, including phosphatidylinositol signaling system, glycerophospholipid metabolism, inositol phosphate metabolism (Table 2) and phosphatidylcholine metabolic process (GO: 0046470) (Additional file 8: Table S6). Additionally, repression of the genes encoding subunits of ATP synthase described above may also result from the repression of Pi deficiency (Zhang et al. 2014).

Based on the above results we compared the expression of genes responsible for root-to-shoot Pi transport between WT and TERF1 tobacco. Phosphate Starvation Response 1 (PHR1), a conserved MYB transcription factor for regulating plant $\mathrm{Pi}$ starvation response, is significantly repressed by TERF1, which can cause the repression of miR399 and miR827 (Fig. 1a) (Chien et al. 2017). Repression of miR399 can upregulate the expression of Phosphate 2 (PHO2) (Fig. 1a), a ubiquitin-conjugating E2 enzyme for degrading Pi transporters phosphate 1 (PHO1) and phosphate transporter 1 (PHT1) (Chien et al. 2017). Moreover, the transcript level of PHO1 is also significantly reduced by TERF1 (Fig. 1a). Repression of miR827 can lead to the upregulation of $N L A$ (Fig. 1a), another ubiquitin E3 ligase for degrading the PHT1 (Chien et al. 2017). So TERF1 represses the expression of the Pi transporters at both transcriptional and posttranslational level, indicating that it can significantly reduce the root-to-shoot Pi uptake. In leaf PHR1 is also significantly repressed by TERF1, which may result from the upregulation of HY5 as it can repress the expression of PHR1 activated by light and ethylene signaling pathway (Fig. 1b) (Liu et al. 2017a, b).

Sulfur assimilation is upregulated by TERF1 as shown by the two GO term, "sulfur compound biosynthetic process" and "sulfur compound metabolic process", are significantly enriched for the upregulated proteins in TERF1 tobacco. The enriched proteins for the sulfur metabolism correlates with the biosynthesis of S-adenosylmethionine (SAM) (No. 2 and No. 143, Additional file 5: Table S3), thiamine (vitamin B1) (No. 158, Additional file 5: Table S3), biotin (vitamin H) (No. 35, Additional file 5: Table S3). Accumulation of SAM provides substrates for the biosynthesis of plant hormones, polyamines, and defense metabolites (Kusano et al. 2010).
Acetyl CoA carboxylase is significantly upregulated by TERF1 (No. 22 and No. 23, Additional file 5: Table S3) and biotin is an important co-factor for it (Nikolau et al. 2003), which contribute to the significant upregulation of fatty acid biosynthesis as shown in Table 1. Biosynthesis of thiamine contributes to the carbon fixation shown in Additional file 7: Table S5, glycolysis and citric acid cycle (Martinis et al. 2016).

Finally we find that TERF1 can increase the supply of one-carbon. Serine accumulation in TERF1 tobacco provides the substrate for one-carbon metabolism. And three serine hydroxymethyltransferase are significantly upregulated by TERF1 (No. 147-149, Additional file 5: Table S3), which convert serine into glycine and 5,10-methylenetetrahydrofolate that are important for plant growth and development (Zhang et al. 2010). And the upregulation of SAM also provide an important source of one-carbon.

\section{TERF1 regulates the retrograde signals in chloroplast}

Retrograde signaling is defined by chan et al. as a process in which a stimulus perturbs chloroplast homeostasis and gives rise to one or more retrograde signals that alter transcriptional through to posttranslational processes and ultimately regulate chloroplast function (Chan et al. 2016).

Transcription as well as protein biosynthesis in chloroplast can act as retrograde signals to regulate the nuclear genes expression, especially the photosynthesisassociated nuclear genes (PhANGs) (Chan et al. 2016). The repression of transcription and protein synthesis in chloroplast by TERF1 described above are important signals for the downregulation of proteins enriched with pathway of "photosynthesis" and "photosynthesisantenna proteins" described in Table 2 and Additional file 6: Table S4 because chloroplast gene expression need to coordinate with their counterparts encoded by nuclear genome performing the same function (Nott et al. 2006).

Methylerythritol cyclodiphosphate (MEcPP), an isoprenoid precursor in chloroplast, is the second retrograde signal regulated by TERF1. TERF1 significantly induces 1-hydroxy-2-methyl-2-(E)-butenyl-4-diphosphate synthase (HDS) (No. 16, Additional file 5: Table S3), which can convert MEcPP into hydroxymethylbutenyl diphosphate (HMBPP) (Chan et al. 2016). The decrease of MEcPP can repress the expression of hydroxyperoxide hyase (HPL) protein (No. 109, Additional file 6: Table S4) and salicylic acid (SA) accumulation (Xiao et al. 2012). HPL protein also correlates with the chloroplast retrograde signal of oxylipins, derived from the oxidized linoleic acid and linolenic acid in chloroplast. The repression of HPL protein will cause the accumulation of 12-oxophytodienoic acid (12-OPDA) as well as 
the biosynthetic precursor for jasmonates (de Souza et al. 2017). Additionally 12-OPDA itself can also act as signal to regulate nuclear gene expression through interaction with the transcription factors binding the TGACG motif, which regulates the stomatal closure in response to drought (de Souza et al. 2017).

Thioredoxins (Trxs) are a large protein family acting as redox regulators to regulate the structure and function of plant proteins through catalyzing the reduction of disulfide bonds in them. (Holmgren 1985). As Trxs can lead to changes in sugars, intermediates of chlorophyll synthesis, ROS, chloroplast protein synthesis, they have been proposed to be involved in the retrograde signaling to nucleus (Brunkard et al. 2015). Two chloroplast-localized Trxs (Trx f1 and HCF164) are significantly repressed by TERF1 (No. 230 and No. 233, Additional file 6: Table S4), while CDSP32 is significantly induced (No. 159, Additional file 5: Table S3). Trx f1 regulates multiple enzymes involved in Calvin-Benson cycle and starch synthesis (Thormahlen et al. 2013). Repression of Trx f1 in TERF1 will attenuate the starch biosynthesis (Thormahlen et al. 2013), indicating that sucrose signaling may be activated by TERF1. HCF164 is involved in the biosynthesis and assembly of cyt b6/f complex (Gabilly et al. 2010), providing an important mechanism for the downregulation of the genes encoding cyt b6/f complex by TERF1 (Additional file 6: Table S4 and Additional file 10: Table S8), indicating that it is involved in the plastid gene expression retrograde signaling. CDSP32 protects photosynthetic apparatus against oxidative damage, showing that it may participate in the retrograde signaling of oxidative stress (Broin et al. 2002).

Tetrapyrroles are the fourth kind of retrograde signals regulated by TERF1. As described above the repression of GluRSAt and PPO2 can inhibit the biosynthesis of tetrapyrroles, which can also lead to the repression of PhANGs (Nott et al. 2006).

The last retrograde signal regulated by TERF1 results from the repression of STN7, which phosphorylates LHCII to mediate state transitions and acts as sensor of the plastoquinol (PQH2)/plastoquinone (PQ) redox state (Chan et al. 2016). PQ/PQH2 redox state can regulate at least 750 nuclear genes expression through STN7 (Chan et al. 2016), so the repression of STN7 partially blocks the redox cues from chloroplast.

\section{Gene set linkage analysis of the DEPs}

Gene set linkage analysis (GSLA) interpretates the differentially expressed genes functional impact on biological processes in Arabidopsis with the aim to solve the problems of "no annotation" or "only conceptually general terms (such as "GO 0007165: signal transduction")" in GO enrichment analysis (Yao et al. 2018). We first searched the corresponding orthologous genes of the
DEPs in Arabidopsis and conducted GSLA analysis. As no significant GO term is enriched for the downregulated proteins, we only analyzed the PPI network of upregulated proteins based on GO enrichment and topology characteristics of the network (Fig. 2).

According to PPI network described in Fig. 2, most of the upregulated DEPs interact with more than 10 proteins, whose functions are summarized in Table 3. These proteins participate in many important biological processes, including salicylic acid signaling pathway, autophagy, chloroplast development, circadian rhythm as well as many important metabolic pathways, including biosynthesis of fatty acid, ethylene, alkaloid, lignin, demonstrating the important roles played by these proteins.

\section{Discussion}

As an important component of ethylene signaling pathway transcription factor of ERF1 plays important roles in activating the ethylene signaling pathway through regulating ethylene responsive genes expression in response to environmental stresses. Our research reveals several new mechanisms for regulating plant response to drought stress by ERF1 protein through regulating chloroplast function.

TERF1 negatively regulates the expression of genes related with photosynthesis

Our results show that overexpression of TERF1 negatively regulates the expression of genes related with photosynthetic light reaction at both transcriptional and translational level, which may result from several important mechanisms. Firstly, our previous research showed that TERF1 significantly repressed the genes encoding the chloroplast transcriptional apparatuses under drought stress condition (Wu et al. 2018). Secondly, a recent study proves that plant response to light needs the degradation of Ethylene-Insensitive3 (EIN3), an upstream activator for ERF1, by phyB (Liu et al. 2017a, b). So overexpression of TERF1 may constitutively repress plant response to light, leading to the repression of genes encoding PhANGs, as it acts downstream of EIN3. Thirdly, TERF1 has also been found to activate the ABA signaling pathway (Zhang et al. 2005) and induce MPK6 (Additional file 11: Figure S3), which also contribute to the repression of the genes related with photosynthetic light reaction (Yamburenko et al. 2015). Finally, the upregulation of carbon fixation and repression of $\mathrm{Pi}$ uptake can also lead to the repression of PhANGs (Hausler et al. 2014; Zhang et al. 2014).

Under stress condition the repression of PhANGs in light will lead to the accumulation of reactive oxygen species (ROS), which can induce the expression of stressrelated genes ( $\mathrm{Su}$ et al. 2018). The interaction between 


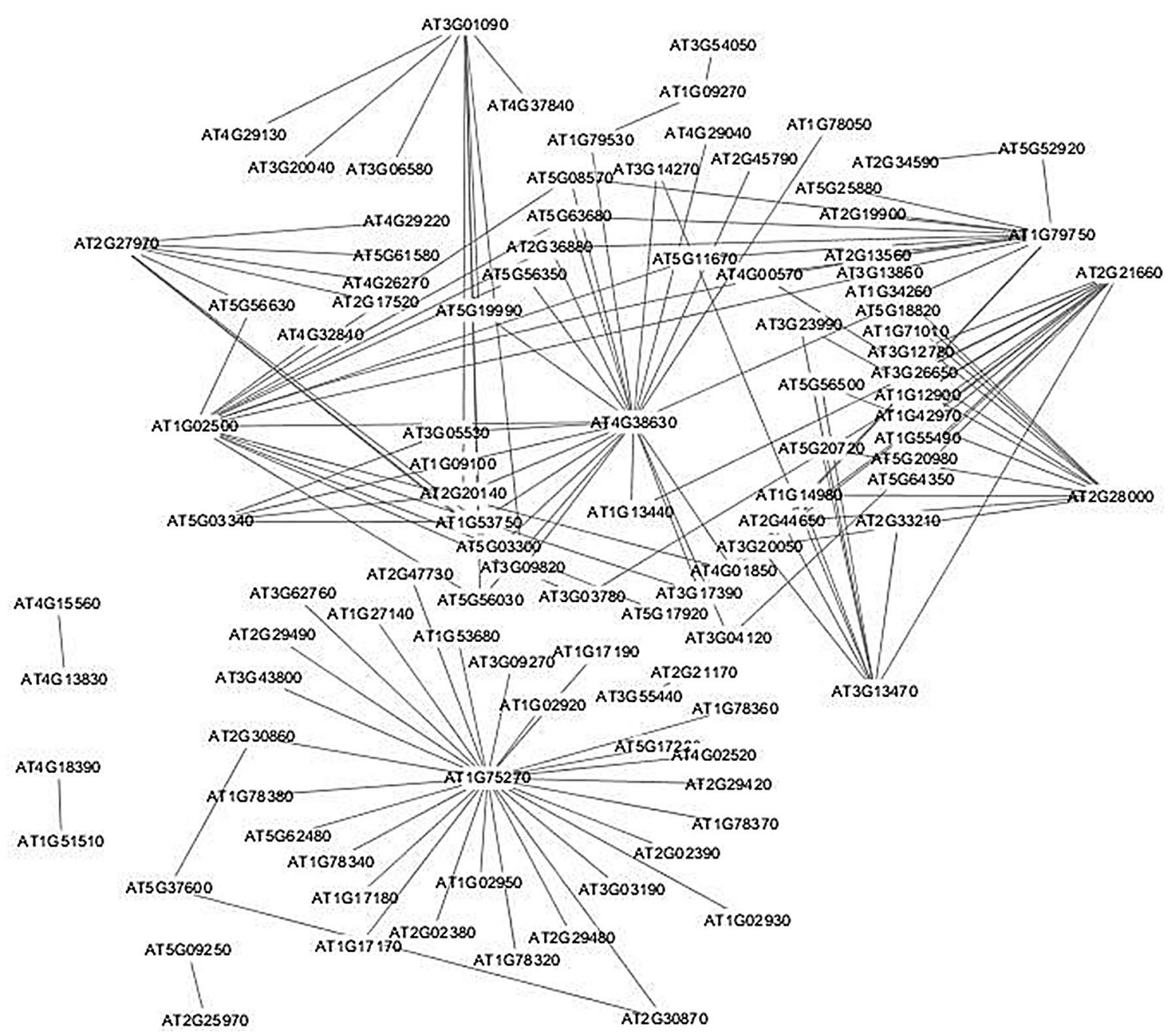

Fig. 2 Protein-protein interaction network of the upregulated DEPs

ROS production and repression of photosynthesisrelated genes orchestrate the trade-off between plant growth and stress tolerance, which can improve plant tolerance of environmental stress (Su et al. 2018).

Photosynthetic genes are encoded by nulear genome and chloroplast genome together. We compared the chloroplast genes expression at both transcriptional level and translational level. Although most of the genes encoded by chloroplast genome are repressed, the correlation between transcript level and protein level is poor. It is reported that translation regulation plays more important roles in chloroplast genes expression than transcription regulation because protein levels vary considerably in response to environmental factors even the corresponding mRNA remain constant (Eberhard et al. 2002). In our study we find that several ribosome genes are both repressed at transcriptional and translational level, which represents a key limit factors for the translational repression for the chloroplast genes (Beligni et al. 2004). Different factors regulate translation of chloroplast genes, so how TERF1 regulates this process deserves future research.

\section{TERF1 may regulate the interaction between chloroplast} and mitochondria

It has been recognized that there exists communication between chloroplast and mitochondria (Raghavendra and Padmasree 2003). From the results we find some important clues for the interaction between chloroplast and mitochondria in TERF1 tobacco that may improve plant tolerance of drought stress. (1) Upregulation of the biosynthesis of valine, leucine and isoleucine not only provide important osmolytes under abiotic stress condition but also can promote oxidization of them in mitochondria, which can provide ATP for photosynthetic carbon fixation under environmental stress conditions (Joshi 
Table 3 Function analysis of the proteins with more than 10 interacting partners in the PPI network

\begin{tabular}{|c|c|c|c|c|c|}
\hline Protein node & Gene name & Enriched GO Term & Degree & Function & Reference \\
\hline AT1G75270 & $\begin{array}{l}\text { Dehydroascorbate reduc- } \\
\text { tase } 2 \text { (DHAR2) }\end{array}$ & GO:0009407 & 28 & $\begin{array}{l}\text { activating the salicylic acid pathway; } \\
\text { modulating the redox states of } \\
\text { ascorbate-glutathione cycle }\end{array}$ & $\begin{array}{l}\text { Ball et al. (2004); Noshi et al. } \\
\text { (2017); Rahantaniaina } \\
\text { et al. (2017) }\end{array}$ \\
\hline AT4G38630 & $\begin{array}{l}\text { Multiubiquitin chain bind- } \\
\text { ing protein } 1 \text { (MBP1) }\end{array}$ & $\begin{array}{l}\text { GO:0006458; GO:0006096; } \\
\text { GO:0006090; } \\
\text { GO:0046835; } \\
\text { GO:0046500; } \\
\text { GO:0046086; } \\
\text { GO:0019318 }\end{array}$ & 23 & $\begin{array}{l}\text { autophagic receptor; promoting the } \\
\text { ubiquitin-mediated degradation of } \\
\text { the oxidized proteins degradation }\end{array}$ & Kurepa et al. (2008) \\
\hline AT2G28000 & $\begin{array}{l}\text { Chaperonin } 60 \text { Alpha } \\
\text { (CPN60A) }\end{array}$ & $\begin{array}{l}\text { GO:0006986; GO:0006458 } \\
\text { GO:0051084; GO:0061077 }\end{array}$ & 17 & $\begin{array}{l}\text { chloroplast development; plant } \\
\text { response to abiotic and biotic stress }\end{array}$ & $\begin{array}{l}\text { Ke et al. (2017); Suzuki et al. } \\
\text { (2009) }\end{array}$ \\
\hline AT3G13470 & $\begin{array}{l}\text { Chaperonin } 60 \text { Beta } \\
\text { (CPN60B) }\end{array}$ & & 14 & & \\
\hline AT2G21660 & $\begin{array}{l}\text { Glycine-rich RNA-binding } \\
\text { protein } 7 \text { (GR-RBP7) }\end{array}$ & $\begin{array}{l}\text { GO:0006458; GO:0006096; } \\
\text { GO:0051084; } \\
\text { GO:0050667 } \\
\text { GO:0046500; GO:0019318 } \\
\text { GO:0061077 }\end{array}$ & 14 & $\begin{array}{l}\text { Stress response; innate immune } \\
\text { response; circadian rhythm; flower } \\
\text { time }\end{array}$ & $\begin{array}{l}\text { Fu et al. (2007); Kwak et al. } \\
\text { (2013); Meyer et al. (2017) }\end{array}$ \\
\hline AT1G02500 & $\begin{array}{l}\text { S-adenosylmethionine } \\
\text { synthetase } 1 \text { (SAM1) }\end{array}$ & $\begin{array}{l}\text { GO:0006096; GO:0006090; } \\
\text { GO:0050667; } \\
\text { GO:0046500; } \\
\text { GO:0061077 }\end{array}$ & 14 & $\begin{array}{l}\text { Ethylene biosynthesis; lignin deposi- } \\
\text { tion, alkaloid biosynthesis }\end{array}$ & $\begin{array}{l}\text { Belbahri et al. (2000); Jin } \\
\text { et al. (2017) }\end{array}$ \\
\hline AT1G79750 & $\begin{array}{l}\text { NADP-malic enzyme } 4 \\
\text { (NADP-ME4) }\end{array}$ & $\begin{array}{l}\text { GO:0006458; GO:0006096 } \\
\text { GO:0006090; GO:0046500; } \\
\text { GO:0061077 }\end{array}$ & 12 & Fatty acid biosynthesis & Wheeler et al. (2005) \\
\hline
\end{tabular}

et al. 2010). As TERF1 significantly repressed ATPase (Additional file 6: Table S4), upregulation of valine, leucine and isoleucine biosynthesis may provide an alternative way to supplement the energy for carbon fixation and cytosol demand under drought stress condition. (2) A key pathway "oxoacid metabolic process" is enriched for the upregulated DEPs, which has been proven to be correlated with a modified TCA cycle in mitochondrial and provides carbon skeleton for $\mathrm{N}$ assimilation in chloroplast under environmental stress condition (Padmasree et al. 2002). Then how TERF1 regulates mitochondrial function and the communication between chloroplast and mitochondrial deserves future research.

\section{TERF1 regulates plant nutrition utilization to improve plant} tolerance of drought stress

Our study has shown that TERF1 can regulate plant utilization of nutrition, which can contribute the improvement of plant tolerance of environmental stress. Firstly, the upregulation of the genes related with carbon fixation positively regulates plant response to different environmental stresses and promotes plant growth in the following darkness (Prasch et al. 2015; Fernandez et al. 2017). Secondly, the enhancement of $\mathrm{N}$ utilization can improve plant tolerance of environmental stresses and maintain cell membrane stability and leaf tissue integrality (Chang et al. 2016). Finally, the reduction of Pi utilization can repress the expression of PhANGs, which may finally lead to the upregulation of stress-related genes (Su et al. 2018).

\section{TERF1 can regulate nuclear gene expression in more diversified ways}

Previously TERF1 was found to regulate nuclear gene expression through binding the cis-element of DRE and GCC box. Our study reveals new potential mechanisms of TERF1 in regulating nuclear gene expression. Firstly, TERF1 can regulate nuclear gene expression through different retrograde signals derived from chloroplast. We have confirmed the interaction between ethylene signaling and retrograde signaling mediated by TERF1 through Q-PCR method, which regulates the expression of PhANGs, plastid redox-associated nuclear genes (PRANGs), singlet oxygen responsive genes (SORGs) (Wu et al. 2018). These retrograde signals not only regulate nuclear gene expression but also connect with different signaling pathways in response to environmental stress as described above. Secondly, TERF1 can regulate gene expression not only at transcriptional level but also at posttranscriptional level. Our analysis shows that TERF1 can regulate Pi uptake at posttranscriptional level through regulating different miRNA. The detailed mechanism of TERF1 regulating nuclear gene expression at posttranscriptional level deserves detailed research in the 
future, which can enrich our knowledge about ethylene signaling pathway.

\section{Conclusions}

In this study we compared the chloroplast proteome between WT and TERF1 tobacco under drought stress condition when the RWC of WT tobacco was significantly lower than the transgenic tobacco. Our results showed significant difference on the proteins related with photosynthesis, carbon fixation, nutrition utilization between WT and TERF1 tobacco, which provide some new insights into the function of TERF1 and ethylene signaling pathway. This proteome analysis also partially confirmed our previous report about the retrograde signaling mediated by TERF1 (Wu et al. 2018), which is a new mechanism for ERF1 protein regulating nuclear gene expression. We also provide new clues for further research on ethylene signaling and ERF1 protein.

\section{Materials and methods}

Plant materials and drought stress treatment

Nicotiana tabacum L. cv. NC89 was used for Agrobacterium-mediated transformation with construct of PROKII containing the ORF of TERF1 driven by 35S promoter (Wu et al. 2018).

Transgenic and WT seeds were first germinated in Petri dish with a moist filter paper at the bottom. After germination seedlings with unanimous growth status were selected and transferred to the soil. The growth condition for the plant is as followings: $28^{\circ} \mathrm{C}$ during day time $\left(300 \mu \mathrm{mol} \mathrm{m}{ }^{-2} \mathrm{~s}^{-1}\right)$ for $16 \mathrm{~h}, 23^{\circ} \mathrm{C}$ at night time for $8 \mathrm{~h}$. All WT and transgenic tobaccos were kept in the same pot to keep unanimous growth condition. Natural dehydration stress was applied for WT and transgenic tobacco at 30 days after germination and relative water content was measured. After 20 days there was significant difference between WT and transgenic tobacco (Additional file 1: Figure S1) and leaves and roots were harvested for further proteome and gene expression analysis. The latest growing leaves were harvested for analysis to minimize the growth difference owing to the drought stress. WT tobaccos under drought stress condition were utilized as control. In this study the proteome and gene expression analysis was applied with three replicates for WT and transgenic tobacco.

\section{Chloroplast isolation and protein extraction}

About $10 \mathrm{~g}$ leaves were grinded in $100 \mathrm{~mL}$ ice cold grinding medium [50 m MHEPES-KOH ( $\mathrm{pH} 8.0), 330 \mathrm{mM}$ sorbitol, $2 \mathrm{mM}$ EDTA- $\mathrm{Na}_{2}$ (pH 8.0), $5 \mathrm{mM}$ ascorbic acid, $5 \mathrm{mM}$ cysteine, $0.05 \%$ bovine serum albumin (BSA)]. Filter the homogenate through a double layer of nylon cloth $(22 \mu \mathrm{m})$. Collect crude chloroplasts by centrifugation for
$3 \mathrm{~min}$ at $1300 \mathrm{~g}$. Resuspend the crude chloroplast pellet in wash medium [50 mM HEPES-KOH (pH 8.0), $330 \mathrm{mM}$ sorbitol, $2 \mathrm{mM}$ EDTA- $\mathrm{Na}_{2}$ (pH 8.0)] by swirling the suspension. Load the resuspended chloroplast on a Percoll step cushion [PF Percoll (40\% or $85 \%), 0.5 \mathrm{mM}$ EDTA, $50 \mathrm{mM}$ HEPES-KOH (pH 8.0), $330 \mathrm{mM}$ sorbitol] and spin for $10 \mathrm{~min}$ at $3750 \mathrm{~g}$ in a swing-out rotor. Collect the intact chloroplasts from the $40 / 85 \%$ Percoll interface using a pipet and add wash medium to dilute the Percoll. Spin the chloroplasts for $3 \mathrm{~min}$ at $1200 \mathrm{~g}$. Remove the supernatant. The pellets are intact chloroplasts.

\section{Chloroplast protein extraction}

Suspend the chloroplast samples in protein extraction buffer (1.52 $\mathrm{g}$ thiourea, $0.3 \mathrm{~g} 3$-[(cholamidopropyl) dimethylammonio]-1-propanesulfonate (CHAPS), $2 \mathrm{~mL}$ water, $1 \mathrm{~mL} 1 \mathrm{M}$ Tris- $\mathrm{HCl}$ (pH 8.0), $2 \mathrm{~mL} \mathrm{50 \%} \mathrm{glyc-}$ erol, $0.2 \mathrm{~mL}$ Triton X-100, $0.2 \mathrm{~mL} 1 \mathrm{M}$ DTT, $3.75 \mathrm{~mL}$ $8 \mathrm{M}$ urea) and sonicate. Centrifuge the homogenate at $12,000 \mathrm{~g}$ for $5 \mathrm{~min}$ at $4{ }^{\circ} \mathrm{C}$ and transfer the supernatant to a new tube. Add 1/10 volume of $100 \%$ trichloroacetic acid (TCA) to the supernatant and mix. Incubate the solution on ice for $15 \mathrm{~min}$. Centrifuge the homogenate at $10,000 \mathrm{~g}$ for $5 \mathrm{~min}$ at $4{ }^{\circ} \mathrm{C}$ and discard the supernatant. Add $1 \mathrm{~mL}$ ice-cold acetone to the pellet and mix. Centrifuge the homogenate at $10,000 \mathrm{~g}$ for $10 \mathrm{~min}$ at $4{ }^{\circ} \mathrm{C}$. Discard the supernatant and resuspend it in $20 \mu \mathrm{L}$ dissolution buffer and $1 \mu \mathrm{L}$ denaturant (2\% SDS) supplied by iTRAQ kit.

\section{ITRAQ labeling and strong fractionation by cation exchange chromatography}

Add iTRAQ labeling reagents to each protein sample and incubate for $1 \mathrm{~h}$ at $37^{\circ} \mathrm{C}$. A $100 \mu \mathrm{L}$ aliquot of water was added to stop the labeling reaction. A $1 \mu \mathrm{L}$ aliquot of sample was removed from each group to test labeling and extraction efficiency, and the sample was subjected to a matrix assisted laser desorption ionization procedure after Ziptip desalting. The six sample groups were pooled and vacuum-dried. Each pool of mixed peptides was lyophilized and dissolved in solution A [ $2 \%$ acetonitrile and $20 \mathrm{mM}$ ammonium formate ( $\mathrm{pH}$ 10.0)]. Then, the samples were loaded onto a reverse-phase column (Luna C18, $4.6 \times 150 \mathrm{~mm}$; Phenomenex, Torrance, CA, USA) and eluted using a step linear elution program: $0-10 \%$ buffer B [ $500 \mathrm{mM} \mathrm{KCl}, 10 \mathrm{mM} \mathrm{KH}_{2} \mathrm{PO}_{4}$ in $25 \%$ acetonitrile, $(\mathrm{pH}$ 2.7)] for $10 \mathrm{~min}, 10-20 \%$ buffer $B$ for $25 \mathrm{~min}, 20-45 \%$ buffer B for $5 \mathrm{~min}$, and $50-100 \%$ buffer B for $5 \mathrm{~min}$ at a flow rate of $0.8 \mathrm{~mL} / \mathrm{min}$. The samples were collected each min and centrifuged for 5-45 min. The fractions (about 40) collected were finally combined into 10 pools and desalted on $\mathrm{C} 18$ Cartridges (Empore ${ }^{\mathrm{TM}}$ standard density 
SPE C18 Cartridges, bed I.D. 7 mm, 3 mL volume; Sigma, St. Louis, MO, USA).

\section{LC-electrospray ionization-MS/MS analysis}

The reconstituted peptides were analyzed with the Q-Exactive mass spectrometer (Thermo Fisher Scientific, Waltham, MA, USA) coupled with a nano highperformance liquid chromatography system (UltiMate 3000 LC Dionex, Thermo Fisher Scientific). The peptides were loaded onto a C18-reversed phase column $(3 \mu \mathrm{m}-\mathrm{C} 18 \mathrm{resin}, 75 \mu \mathrm{m} \times 15 \mathrm{~cm})$ and separated on an analytical column $(5 \mu \mathrm{m} \mathrm{C} 18$ resin, $150 \mu \mathrm{m} \times 2 \mathrm{~cm}$; Dr. Maisch $\mathrm{GmbH}$, Ammerbuch, Germany) using mobile phase A: $0.5 \%$ formic acid $[\mathrm{FA}] / \mathrm{H}_{2} \mathrm{O}$ and $\mathrm{B}: 0.5 \% \mathrm{FA} /$ $\mathrm{ACN}$ at a flow rate of $300 \mathrm{~nL} / \mathrm{min}$, using a $150 \mathrm{~min}$ gradient. Spectra were acquired in data-dependent mode. The 10 most intense ions selected for MS scanning $(300-1800 \mathrm{~m} / \mathrm{z}, 60,000$ resolution at $\mathrm{m} / \mathrm{z} 400$, accumulation of $1 \times 106$ ions for a maximum of $500 \mathrm{~ms}, 1$ microscan). The isolation window was $1.3 \mathrm{~m} / \mathrm{z}$, and the MS/MS spectra were accumulated for $150 \mathrm{~ms}$ using an Orbitrap. MS/MS spectra were measured at resolution of 15,000 at $\mathrm{m} / \mathrm{z} 400$. Dynamic precursor exclusion was allowed for $120 \mathrm{~s}$ after each MS/MS spectrum measurement and was set to 17,500 at $\mathrm{m} / \mathrm{z} 200$. Normalized collision energy was $30 \mathrm{eV}$ and the underfill ratio, which specifies the minimum percentage of the target value likely to be reached at the maximum fill time, was defined as $0.1 \%$. The instrument was run with peptide recognition mode enabled.

\section{Sequence database search}

The raw mass data were processed for the peptide data analysis using Proteome Discoverer 1.4 (ver.1.4.0.288; Thermo Fisher Scientific) with a false discovery rate $[\mathrm{FDR}=\mathrm{N}($ decoy $) * 2 /((\mathrm{N}($ decoy $)+\mathrm{N}($ target $)))<1 \%$ and expected cutoff or ion score $<0.05$ (with $95 \%$ confidence) for searching the Uniprot Nicotiana tobacco Complete Proteome database. Protein probabilities were assigned using the Protein Prophet algorithm, and proteins with at least two unique peptides were identified. The following options were used to identify the proteins: peptide mass tolerance $= \pm 15 \mathrm{ppm}$, fragment mass tolerance $=20$ $\mathrm{mmu}, \quad$ enzyme $=$ trypsin, $\max$ missed cleavage $=2$, fixed modification: carbamidomethyl (C), iTRAQ8plex (N-term), iTRAQ8plex (K), iTRAQ8plex (Y), variable modification: Oxidation (M), Acetyl (Protein N-term).

\section{RNA isolation and reverse transcription}

Total RNA and miRNA were extracted from about $200 \mathrm{mg}$ leaf and $500 \mathrm{mg}$ root using RNAiso Plus Kit and RNAiso for small RNA kit (Takara Biomedical Technology, Japan), respectively. RNA integrity was confirmed by electrophoresis and RNA quantity was determined by NanoDrop ND-1000 spectrophotometer (NanoDrop Technologies, Wilmington, DE, USA). Total RNA and miRNA were utilized for reverse transcription using Prime Script ${ }^{\mathrm{TM}}$ RT reagent Kit with gDNA Eraser and Mir- $\mathrm{X}^{\mathrm{TM}}$ miRNA First Strand Synthesis Kit (Takara Biomedical Technology, Japan), respectively.

\section{Quantitative real-time polymerase chain reaction (qRT-PCR)}

qRT-PCR was performed with 3 replicates by CFX 96 real-time PCR detection system (Bio-Rad, Hercules, USA). SYBR ${ }^{\circledR}$ Premix Ex Taq ${ }^{\text {TM }}$ II (Tli RNaseH Plus) kit and Mir- $\mathrm{X}^{\mathrm{TM}}$ miRNA qRT-PCR SYBR ${ }^{\circledR}$ Kit (Takara Biomedical Technology, Japan) was used for mRNA and miRNA qRT-PCR, respectively. Tobacco L25 (GenBank

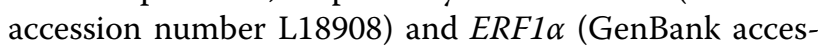
sion number AF120093) were utilized to normalize the mRNA genes expression value. 5S RNA was utilized to normalize the miRNA genes expression value. Beacon designer 8.0 was utilized to design the gene specific primers (Additional file 12: Table S1). The qPCR reaction was performed as following: $95^{\circ} \mathrm{C}$ for $5 \mathrm{~min}$, followed by 40 cycles $\left(95^{\circ} \mathrm{C}\right.$ for $30 \mathrm{~s}, 60^{\circ} \mathrm{C}$ for $30 \mathrm{~s}$, and $72{ }^{\circ} \mathrm{C}$ for $30 \mathrm{~s}$ ), followed by melting curve analysis: at $50^{\circ} \mathrm{C}$ for $30 \mathrm{~s}$ and then at $65-95{ }^{\circ} \mathrm{C}\left(0.5{ }^{\circ} \mathrm{C}\right.$ increments, $5 \mathrm{~s}$ for each). The chloroplast genome sequences of tobacco were retrieved from NCBI database (Accession number: NC_001879.2). The nuclear-encoded genes sequences of tobacco were retrieved from Sol Genomics Network database according to the latest genome annotation (https://solgenomic s.net/).

\section{Relative water content analysis}

Relative water content (RWC) was calculated by the formula: $\mathrm{RWC}(\%)=(\mathrm{FW}-\mathrm{DW}) /(\mathrm{TW}-\mathrm{DW}) \times 100$. FW: fresh weight, TW: turgid weight, DW: dry weight.

\section{Additional files}

Additional file 1: Figure S1. Comparison of relative water content (RWC) under drought stress condition.

Additional file 2: Table S9. Chloroplast proteins identified in WT and TERF1 tobacco by iTRAQ method.

Additional file 3: Table S2. Identification and quantification of chloroplast proteins in WT and TERF1 tobacco.

Additional file 4: Figure S2. Principal component analysis of the chloroplast proteome in WT and TERF1 tobacco.

Additional file 5: Table S3. Proteins up regulated by TERF1 under drought stress condition. 
Additional file 6: Table S4. Proteins downregulated by TERF1 under drought stress condition.

Additional file 7: Table S5. Gene ontology enrichment analysis for the upregulated proteins by TERF1.

Additional file 8: Table S6. Gene ontology analysis for the downregulated proteins by TERF1.

Additional file 9: Table S7. Identification and quantification of the chloroplast genome encoded proteins in WT and TERF1 tobacco.

Additional file 10: Table S8. Expression analysis of the chloroplast genome encoded genes.

Additional file 11: Figure S3. Expression analysis of MPK6 between WT and TERF1 tobacco. Means \pm SDs, $n=3 ;{ }^{*}$ - significantly different at $5 \%$ level of probability.

Additional file 12: Table S1. Primers Used in This Research.

\section{Abbreviations}

ABA: abscisic acid; DEPs: differentially expressed proteins; ERF: ethylene response factor; GO: gene ontology; GS: glutamine synthetase; GSLA: gene set linkage analysis; N: nitrogen; NR: nitrate reductase; Pi: phosphorus; PPI: protein-protein interaction; RWC: relative water content; TERF1: tomato ethylene responsive factor 1 ; Trx: thioredoxin; WT: wild type.

\section{Authors' contributions}

YY conceived the concept, designed the experiment and provide revisions for the manuscript. WW performed the experiments, analyzed the data and wrote the manuscript. Both authors read and approved the final manuscript.

\section{Acknowledgements}

We are grateful to Dr. Rongfeng Huang for providing the construct of TERF1.

\section{Competing interests}

The authors declare that they have no competing interests.

\section{Availability of data and materials}

The mass spectrometry proteomics data have been deposited to the iProX Integrated Proteome Resources (http://www.iprox.org/page/HMV006.html) with the dataset identifier <|PX0001206000>.

\section{Consent for publication}

Authors agree to the terms of the Springer Open Copyright and License Agreement.

\section{Ethics approval and consent to participate}

Not applicable.

\section{Funding}

This study was supported by grants from Chinese Academy of Agricultural Sciences Basal Research Fund (No. 1610042018006).

\section{Publisher's Note}

Springer Nature remains neutral with regard to jurisdictional claims in published maps and institutional affiliations.

Received: 19 July 2018 Accepted: 17 October 2018 Published online: 29 October 2018

\section{References}

Ball L, Accotto GP, Bechtold U, Creissen G, Funck D, Jimenez A, Kular B, Leyland N, Mejia-Carranza J, Reynolds H, Karpinski S, Mullineaux PM (2004) Evidence for a direct link between glutathione biosynthesis and stress defense gene expression in Arabidopsis. Plant Cell 16:2448-2462

Belbahri L, Chevalier L, Bensaddek L, Gillet F, Fliniaux MA, Boerjan W, Inze D, Thomas D, Thomasset B (2000) Different expression of an
S-adenosylmethionine synthetase gene in transgenic tobacco callus modifies alkaloid biosynthesis. Biotechnol Bioeng 69:1 1-20

Beligni MV, Yamaguchi K, Mayfield SP (2004) The translational apparatus of Chlamydomonas reinhardtii chloroplast. Photosynth Res 82(3):315-325

Broin M, Cuine S, Eymery F, Rey P (2002) The plastidic 2-cysteine peroxiredoxin is a target for a thioredoxin involved in the protection of the photosynthetic apparatus against oxidative damage. Plant Cell 14:1417-1432

Brunkard JO, Runkel AM, Zambryski PC (2015) Chloroplasts extend stromules independently and in response to internal redox signals. Proc Natl Acad Sci USA 112:10044-10049

Chan KX, Phua SY, Crisp P, Mcquinn R, Pogson BJ (2016) Learning the languages of the chloroplast: retrograde signaling and beyond. Annu Rev Plant Biol 67:25-53

Chang Z, Liu Y, Dong H, Teng K, Han L, Zhang X (2016) Effects of cytokinin and nitrogen on drought tolerance of creeping bentgrass. PLOS ONE 11(4):e154005

Chien PS, Chiang CB, Wang Z, Chiou TJ (2017) MicroRNA-mediated signaling and regulation of nutrient transport and utilization. Curr Opin Plant Biol 39:73-79

Daniell H, Lin CS, Yu M, Chang WJ (2016) Chloroplast genomes: diversity, evolution, and applications in genetic engineering. Genome Biol 17:134

de Souza A, Wang JZ, Dehesh K (2017) Retrograde signals: integrators of interorganellar communication and orchestrators of plant development. Annu Rev Plant Biol 68:85

Eberhard S, Drapier D, Wollman FA (2002) Searching limiting steps in the expression of chloroplast-encoded proteins: relations between gene copy number, transcription, transcript abundance and translation rate in the chloroplast of Chlamydomonas reinhardtii. Plant J 31(2):149-160

Fernandez O, Ishihara H, George GM, Mengin V, Flis A, Sumner D, Arrivault S, Feil R, Lunn JE, Zeeman SC, Smith AM, Stitt M (2017) Leaf starch turnover occurs in long days and in falling light at the end of the day. Plant Physiol 174:2199-2212

Filleur S, Dorbe MF, Cerezo M, Orsel M, Granier F, Gojon A, Daniel-Vedele F (2001) An arabidopsis T-DNA mutant affected in Nrt2 genes is impaired in nitrate uptake. FEBS Lett 489:220-224

Fu ZQ, Guo M, Jeong BR, Tian F, Elthon TE, Cerny RL, Staiger D, Alfano JR (2007) A type III effector ADP-ribosylates RNA-binding proteins and quells plant immunity. Nature 447:284-288

Gabilly ST, Dreyfuss BW, Karamoko M, Corvest V, Kropat J, Page MD, Merchant SS, Hamel PP (2010) CCS5, a thioredoxin-like protein involved in the assembly of plastid c-type cytochromes. J Biol Chem 285:29738-29749

Galvez S, Lancien M, Hodges M (1999) Are isocitrate dehydrogenases and 2-oxoglutarate involved in the regulation of glutamate synthesis? Trends Plant Sci 4:484-490

Glass F, Hartel B, Zehrmann A, Verbitskiy D, Takenaka M (2015) MEF13 requires MORF3 and MORF8 for RNA editing at eight targets in mitochondrial mRNAs in Arabidopsis thaliana. Molecular Plant 8:1466-1477

Hausler RE, Heinrichs L, Schmitz J, Flugge UI (2014) How sugars might coordinate chloroplast and nuclear gene expression during acclimation to high light intensities. Mol Plant 7:1121-1137

Holmgren A (1985) Thioredoxin. Annu Rev Biochem 54:237-271

Huang Z, Zhang Z, Zhang X, Zhang H, Huang D, Huang R (2004) Tomato TERF1 modulates ethylene response and enhances osmotic stress tolerance by activating expression of downstream genes. FEBS Lett 573:110-116

Huang C, Yu QB, Li ZR, Ye LS, Xu L, Yang ZN (2017) Porphobilinogen deaminase HEMC interacts with the PPR-protein AtECB2 for chloroplast RNA editing. Plant J 92:546-556

Jin Y, Ye N, Zhu F, Li H, Wang J, Jiang L, Zhan J (2017) Calcium-dependent protein kinase CPK28 targets the methionine adenosyltransferases for degradation by the 265 proteasome and affects ethylene biosynthesis and lignin deposition in Arabidopsis. Plant J 90:304-318

Joshi V, Joung JG, Fei Z, Jander G (2010) Interdependence of threonine, methionine and isoleucine metabolism in plants: accumulation and transcriptional regulation under abiotic stress. Amino Acids 39:933-947

Kato Y, Miura E, Ido K, Ifuku K, Sakamoto W (2009) The variegated mutants lacking chloroplastic FtsHs are defective in D1 degradation and accumulate reactive oxygen species. Plant Physiol 151:1790-1801 
Kavas M, Kizildogan A, Gokdemir G, Baloglu MC (2015) Genome-wide investigation and expression analysis of AP2-ERF gene family in salt tolerant common bean. Excli J 14:1187-1206

Ke X, Zou W, Ren Y, Wang Z, Li J, Wu X, Zhao J (2017) Functional divergence of chloroplast Cpn60alpha subunits during Arabidopsis embryo development. PLoS Genet 13:e1007036

Kmiecik P, Leonardelli M, Teige M (2016) Novel connections in plant organellar signalling link different stress responses and signalling pathways. J Exp Bot 67:3793-3807

Kosmala A, Perlikowski D, Pawlowicz I, Rapacz M (2012) Changes in the chloroplast proteome following water deficit and subsequent watering in a high- and a low-drought-tolerant genotype of Festuca arundinacea. J Exp Bot 63:6161-6172

Kurepa J, Toh-E A, Smalle JA (2008) 26S proteasome regulatory particle mutants have increased oxidative stress tolerance. Plant Journal 53:102-114

Kusano M, Fukushima A, Redestig H, Kobayashi M, Otsuki H, Onouchi H, Naito S, Hirai MY, Saito K (2010) Comparative metabolomics charts the impact of genotype-dependent methionine accumulation in Arabidopsis thaliana. Amino Acids 39:1013-1021

Kwak KJ, Kang H, Han KH, Ahn SJ (2013) Molecular cloning, characterization, and stress-responsive expression of genes encoding glycine-rich RNAbinding proteins in Camelina sativa L. Plant Physiol Biochem 68:44-51

Li W, Xiang F, Zhong M, Zhou L, Liu H, Li S, Wang X (2017) Transcriptome and metabolite analysis identifies nitrogen utilization genes in tea plant (Camellia sinensis). Sci Rep 7(1):1693

Liu W, Sun Q, Wang K, Du Q, Li WX (2017a) Nitrogen limitation adaptation (NLA) is involved in source-to-sink remobilization of nitrate by mediating the degradation of NRT1.7 in Arabidopsis. New Phytol 214:734-744

Liu X, Liu R, LiY, Shen X, Zhong S, Shi H (2017b) EIN3 and PIF3 Form an interdependent module that represses chloroplast development in buried seedlings. Plant Cell 29:3051-3067

Martinis J, Gas-Pascual E, Szydlowski N, Crevecoeur M, Gisler A, Burkle L, Fitzpatrick TB (2016) Long-distance transport of thiamine (Vitamin B1) is concomitant with that of polyamines. Plant Physiol 171:542-553

Meyer K, Koster T, Nolte C, Weinholdt C, Lewinski M, Grosse I, Staiger D (2017) Adaptation of iCLIP to plants determines the binding landscape of the clock-regulated RNA-binding protein AtGRP7. Genome Biol 18:204

Miller AJ, Fan XR, Shen QR, Smith SJ (2008) Amino acids and nitrate as signals for the regulation of nitrogen acquisition. J Exp Bot 59(1):111-119

Nikolau BJ, Ohlrogge JB, Wurtele ES (2003) Plant biotin-containing carboxylases. Arch Biochem Biophys 414:211-222

Noshi M, Yamada H, Hatanaka R, Tanabe N, Tamoi M, Shigeoka S (2017) Arabidopsis dehydroascorbate reductase 1 and 2 modulate redox states of ascorbate-glutathione cycle in the cytosol in response to photooxidative stress. Biosci Biotechnol Biochem 81:523-533

Nott A, Jung HS, Koussevitzky S, Chory J (2006) Plastid-to-nucleus retrograde signaling. Annu Rev Plant Biol 57:739-759

Padmasree K, Padmavathi L, Raghavendra AS (2002) Essentiality of mitochondrial oxidative metabolism for photosynthesis: optimization of carbon assimilation and protection against photoinhibition. Crit Rev Biochem Mol Biol 37(2):71-119

Prasch CM, Ott KV, Bauer H, Ache P, Hedrich R, Sonnewald U (2015) ss-amylase 1 mutant Arabidopsis plants show improved drought tolerance due to reduced starch breakdown in guard cells. J Exp Bot 66:6059-6067

Raghavendra AS, Padmasree K (2003) Beneficial interactions of mitochondrial metabolism with photosynthetic carbon assimilation. Trends Plant Sci 8(11):546-553

Rahantaniaina MS, Li S, Chatel-Innocenti G, Tuzet A, Issakidis-Bourguet E, Mhamdi A, Noctor G (2017) cytosolic and chloroplastic DHARs cooperate in oxidative stress-driven activation of the salicylic acid Pathway. Plant Physiol 174:956-971

Seung D, Soyk S, Coiro M, Maier BA, Eicke S, Zeeman SC (2015) Protein targeting to starch is required for localising granule-bound starch synthase to starch granules and for normal amylose synthesis in Arabidopsis. PLoS Biol 13:e1002080
Shimada H, Sugiura M (1991) Fine structural features of the chloroplast genome: comparison of the sequenced chloroplast genomes. Nucleic Acids Res 19:983-995

Su J, Yang L, Zhu Q, Wu H, He Y, Liu Y, Xu J, Jiang D, Zhang S (2018) Active photosynthetic inhibition mediated by MPK3/MPK6 is critical to effectortriggered immunity. PLoS Biol 16:e2004122

Sulmon C, Gouesbet G, Ramel F, Cabello-Hurtado F, Penno C, Bechtold N, Couee I, El AA (2011) Carbon dynamics, development and stress responses in Arabidopsis: involvement of the APL4 subunit of ADP-glucose pyrophosphorylase (starch synthesis). PLoS ONE 6:e26855

Suzuki K, Nakanishi H, Bower J, Yoder DW, Osteryoung KW, Miyagishima SY (2009) Plastid chaperonin proteins Cpn60 alpha and Cpn60 beta are required for plastid division in Arabidopsis thaliana. BMC Plant Biol 9:38

Taniguchi M, Taniguchi Y, Kawasaki M, Takeda S, Kato T, Sato S, Tabata S, Miyake H, Sugiyama T (2002) Identifying and characterizing plastidic 2-oxoglutarate/malate and dicarboxylate transporters in Arabidopsis thaliana. Plant Cell Physiol 43:706-717

Thormahlen I, Ruber J, von Roepenack-Lahaye E, Ehrlich SM, Massot V, Hummer C, Tezycka J, Issakidis-Bourguet E, Geigenberger P (2013) Inactivation of thioredoxin $\mathrm{f1}$ leads to decreased light activation of ADP-glucose pyrophosphorylase and altered diurnal starch turnover in leaves of Arabidopsis plants. Plant Cell Environ 36:16-29

Tiller N, Bock R (2014) The translational apparatus of plastids and its role in plant development. Mol Plant 7:1105-1120

Uberegui E, Hall M, Lorenzo O, Schroder WP, Balsera M (2015) An Arabidopsis soluble chloroplast proteomic analysis reveals the participation of the executer pathway in response to increased light conditions. J Exp Bot 66:2067-2077

Wang L, Liang W, Xing J, Tan F, Chen Y, Huang L, Cheng CL, Chen W (2013) Dynamics of chloroplast proteome in salt-stressed mangrove Kandelia candel (L.) Druce. J Proteome Res 12:5124-5136

Wang YY, Cheng YH, Chen KE, Tsay YF (2018) Nitrate transport, signaling, and use efficiency. Annu Rev Plant Biol. 69:85-122

Wheeler MC, Tronconi MA, Drincovich MF, Andreo CS, Flugge UI, Maurino VG (2005) A comprehensive analysis of the NADP-malic enzyme gene family of Arabidopsis. Plant Physiol 139:39-51

Wu W, Liu LL, Yang T, Wang JH, Wang JY, LV P, Yan YC (2018) Gene expression analysis reveals function of TERF1 in plastid-nucleus retrograde signaling under drought stress conditions. Biol Plant. https://doi.org/10.1007/s1053 5-018-0771-x

Xiao Y, Savchenko T, Baidoo EE, Chehab WE, Hayden DM, Tolstikov V, Corwin JA, Kliebenstein DJ, Keasling JD, Dehesh K (2012) Retrograde signaling by the plastidial metabolite MEcPP regulates expression of nuclear stressresponse genes. Cell 149:1525-1535

Yamburenko MV, Zubo YO, Borner T (2015) Abscisic acid affects transcription of chloroplast genes via protein phosphatase $2 \mathrm{C}$-dependent activation of nuclear genes: repression by guanosine-3'-5'-bisdiphosphate and activation by sigma factor 5. Plant J 82:1030-1041

Yao H, Wang X, Chen P, Hai L, Jin K, Yao L, Mao C, Chen X (2018) Predicted Arabidopsis interactome resource and gene set linkage analysis: a transcriptomic analysis resource. Plant Physiol 177(1):422-433

Zhang X, Zhang Z, Chen J, Chen Q, Wang XC, Huang R (2005) Expressing TERF1 in tobacco enhances drought tolerance and abscisic acid sensitivity during seedling development. Planta 222(3):494-501

Zhang X, Szydlowski N, Delvalle D, D'Hulst C, James MG, Myers AM (2008) Overlapping functions of the starch synthases SSII and SSIII in amylopectin biosynthesis in Arabidopsis. BMC Plant Biol 8:96

Zhang Y, Sun K, Sandoval FJ, Santiago K, Roje S (2010) One-carbon metabolism in plants: characterization of a plastid serine hydroxymethyltransferase. Biochem J 430(1):97-105

Zhang K, Liu H, Tao P, Chen H (2014) Comparative proteomic analyses provide new insights into low phosphorus stress responses in maize leaves. PLoS ONE 9(5):e98215

Zubo YO, Yamburenko MV, Kusnetsov VV, Borner T (2011) Methyl jasmonate, gibberellic acid, and auxin affect transcription and transcript accumulation of chloroplast genes in barley. J Plant Physiol 168:1335-1344 